\title{
DE LA SURPRISE, TOUJOURS DE LA SURPRISE. DU TEMPS, TOUJOURS PLUS DE TEMPS...
}

\author{
DE LA SORPRESA, SIEMPRE DE LA SORPRESA. \\ DEL TIEMPO, SIEMPRE MÁS TIEMPO...
}

Natalie Depraz

Université de Rouen Normandie / Archives-Husserl ENS, Paris natalie.depraz@univ-rouen.fr

About More Phenomenology of Time

(FLORIDA, DELRAY BEACH, 15-20 NOVEMBRE 1995)

(EXTRAIt DE PolyLOGUes)

15 novembre, 11h45: départ pour Miami. Neuf heures de vol, six heures de décalage, à bord d'un Boing 747. Tout change de dimension: il y a plusieurs accès à bord, en fonction du numéro de notre place, les consignes de sécurité sont projetées sur grand écran, et tout y est montré, même l'évacuation par toboggan, l'amerrissage et les canots de sauvetage... Nous volerons longtemps. Je n'ai jamais passé un temps aussi long dans un avion: je ne pars pas très longtemps — six jours- mais je pars loin. Cette fois, c'est l'éloignement spatial, plus que la longueur du séjour dans le temps, qui crée nostalgie et émotion. Je n'ai jamais connu cela. C'est la première fois que je me sens si démunie. 
15h: en vol. Nous volons à 682 kilomètres par heure et la température est de 58 degrés celsius.

19h: il est 1h p.m. à Miami, où nous arriverons dans deux heures. Le temps passe sans passer, puisque nous le remontons: nous sommes en pleine phénoménologie génétique. Suspendue dans l'air, je suis comme en apesanteur. Il n'y a plus ni temps, ni espace connus, et pourtant, je reste un individu parmi d'autres: il y a bien un autre temps, celui que rythment repas, collations, films et informations, un autre lieu, clos et climatisé, partagé. Font-ils de moi cet individu qui ressent ce que je suis en train d'écrire? Il semble que je sois cet individu-ci par delà tel temps et tel espace. Voilà qui est à présent plus que manifeste.

3h 45 p.m. à Miami (21h45 à Paris). Il fait 22 degrés celsius, température inhabituellement basse pour la saison. J'attends parmi indiens, noirs et hispanisants le shuttle qui nous emmènera à la gare; puis, le fameux Trirail, archaïque, poussif et bigarré m'emporte vers Delray Beach, traversant zone sur zone. Suis-je bien aux Etats-Unis?

5h 30: Magnifique coucher de soleil sur les palmiers, rouge et dense sur fond de bleu profond.

5h 45: arrivée à Delray Beach. Lester Embree m'y attend. Son aspect «cowboy», sur lequel insistait en souriant Rudolf Bernet avant mon départ, me frappe moins que sa simplicité. Sa maison ornée de fines colonnes qui rappellent le style colonial date de 1920: son «ancienneté» est remarquable, par rapport aux maisons le plus souvent construites dans les années 80. Delray Beach a 120 ans, elle reste implicitement séparée en deux quartiers, I'un noir, l'autre blanc. Nous allons manger dans un restaurant mexicain, très typique à l'intérieur: de l'extérieur, on dirait un motel! Je goûte à différents plats très «spicy» en buvant de la sangria au fil d'une conversation qui passe de l'Inde à Cuba, puis à l'écologie et à la politique chez Husserl, pour enfin déboucher sur celle de Simone Weil...

Couchée à 3 heures du matin (H. F.) -9 h p. m. - je dors d'une traite jusqu'à 6 h a. $\mathrm{m}$. (12 heures en France): le lever du soleil, à 6 heures 15, est de toute beauté.

16 novembre, 15h-17h: longue marche sur la plage. L'océan est doux et chaud. Je t'imagine près de moi, à marcher sur le sable, et nous allons pendant des heures, le soleil devant nous, Jean-Emmanuel gambadant au devant de nous. Il n'y a personne, et la plage s'étend à perte de vue.

Au début, je cherche désespérément des galets, puis je découvre mieux que cela, des pierres incrustées de coquillages minuscules et, plus encore, de magnifiques coquillages doux au toucher, roses et jaunes. Le vent souffle, mais il fait tiède. Je vais de l'avant, à 
la recherche de l'immensité. Grand silence. Seul le fracassement des vagues sur la plage l'interrompt et fait écho à cette immensité. Que dire d'autre, sinon que bonheur d'être là et tristesse profonde que tu ne sois pas là s'affrontent en moi, s'entrechoquent jusqu'à la souffrance, jusqu'à l'apaisement. Tu es présent dans chacun de mes pas, dans chacune de mes impressions, de mes pensées. Ensemble, tous les trois, nous irons en Floride.

Ce soir, nous dînerons chez Lester Embree, dans cette étrange maison qui n'a pas de foyer, pas d'intimité, mais qui respire en son sein l'air du dehors, des arbres, de l'herbe et de l'air. Elle va si bien avec cette phrase qu'il m'a dite ce matin, pour me donner à comprendre son souci de l'écologie: «My home is a jungle, the jungle is my home». C'est un partisan farouche de la «deep ecology»: ses articles comme ses cours en témoignent. Ethnicity, gender, environment sont ses trois catégories-clé. Hostile aux analyses textuelles, il revendique une pratique vivante et quotidienne de la phénoménologie. Je suis curieuse de voir comment les journées sur le temps vont se dérouler: il hait le gazon et les chats domestiqués.

Quant à Atlantic Florida University, c'est un mélange étonnant de béton et de verdure. Les bâtiments sont sinistres et, tout autour, les gazons et les palmiers «rappellent»la nature. Brave New World n'est pas loin.

En parcourant le 1995 Visitor's Guide du Seagate Hotel, Private Club où je dispose d'une suite très luxueuse, avec télévision, cuisine, salle de bain, salon et chambre double (Franck n'est pas auprès de moi!), je découvre sept différentes Houses of Worship: 6 Catholic Churches, 3 Episcopal Churches, 3 Presbyterian Churches, 3 Methodist, 1 Baptist, 1 Lutheran and 3 Synagogues... Je suis stupéfaite.

Il est 17 heures et le soleil se couche lentement sur les palmiers. Je me souviens brutalement d'une remarque de Lester Embree sur l'orientation ou, plutôt, sur l'absence d'orientation dans ce plat pays qu'est la Floride (dans la South Florida, on ne gagne que deux mètres en 25 kms!): puisque l'on ne peut s'orienter par rapport à la terre, orientons-nous par rapport au ciel, et au mouvement des nuages!

17 novembre: première journée de travail. John Brough ouvre la Conference avec un exposé remarquable sur les différentes modalités de temporalité à l'oeuvre dans les arts plastiques et, notamment, dans la peinture. Mais la question reste ouverte de savoir si la «plasticité» du temps dans les arts picturaux est telle (son titre est «Plastic Time») qu'aucune structure temporelle intrinsèque ne reste dominante ou ne soit un fil conducteur pour la compréhension de l'oeuvre plastique. J'essaie devant lui de défendre l'idée d'un nécessaire critère unificateur de la temporalité d'une peinture donnée, à savoir son caractère synoptique (i.e. simultané), mais il ne semble pas convaincu. 
Suit un exposé typiquement New Age où les références au bouddhisme, à Gurtchiev et à Husserl se côtoient, suggérant la présence d'une mindfulness révélée par des «altered states of consciousness»: I'air du temps est là au Seagate Hotel.

Les autres exposés sont sans intérêt: R. Bruzina «sticks to the text», les autres parlent d'historicité et de narrativité. Dans la conversation avec K. Held qui suit l'exposé New Age, nous avons une confrontation intéressante: il insiste sur la différence irréductible entre l'unité plotinienne régissant en dernière instance l'ensemble de la théologie occidentale, laquelle reconduit inévitablement la figure onto-théo-logique de la métaphysique, et l'indifférenciation an-égoïque qui traverse le bouddhisme Zen. Il réduit ce faisant la théologie occidentale à un mysticisme fusionnel de l'unité et ne saisit pas la portée d'une voie négative ou gnostique qui déferait de l'intérieur le cadre même de I'onto-théo-logie; il accentue par ailleurs l'opposition avec l'Orient, lors même que la méditation bouddhiste n'abolit pas purement et simplement l'ego, mais le resitue plutôt à une place relative.

17h 30: le travail est fini. Bain de mer crépusculaire en compagnie de Klaus Held et de Tom Nenon. Le ciel est plus que nuageux, le vent souffle et l'océan est couleur gris-acier, tumultueux et violent: les vagues manquent à chaque instant de nous engloutir. Mais l'eau est quasiment tiède. Je me laisse tout d'abord gagner par la chaleur, par l'aisance de la nage, par la fluidité de l'océan. Soudain, une angoisse sourde m'étreint. Je n'ai pas pied, les vagues sont monstrueuses, ma respiration haletante. Je regagne la plage, où K. Held me retrouve peu après... Il fait bon: le vent est doux. T. Nenon nous y rejoint aussi. Il y eut cette sensation intense de lutte -vaine- contre l'élément marin.

18 novembre: c'est aujourd'hui que je parle. Contrairement à d'habitude, je n'ai pas le trac. Bien que mon texte ne soit qu'une esquisse, bien que mon anglais soit loin d'être parfait, j'ai l'intime conviction que la piste que je déploie est juste. Mon paper s'intitule: «Hyletic Absolute Flow and World Creation».

La discussion est très animée. Lester Embree a l'air plus que satisfait. Klaus Held, John Brough, Ronald Bruzina, mais aussi Tom Nenon cherchent à prolonger le parcours. C'est passionnant. Je me sens très à l'aise. Klaus Held pose la question de la pertinence du maintien de l'opposition de la matière et de forme, et plaide pour l'unité. Je réponds en valorisant la non-dualité des deux, et la nécessité de ne pas inventer de nouveaux termes pour dire un inédit conceptuel qui est aussi reprise altérée de l'ancien. Je crois que je n'ai jamais été aussi à l'aise. Un certain Simon reprend la question de Held en défendant l'idée selon laquelle on ne peut, selon lui, sortir de l'opposition du monisme et du dualisme, celle-ci étant par ailleurs inféconde philosophiquement, et reconduisant toujours à un monisme déguisé. Ayant déjà expliqué clairement le statut de la nondualité, je réponds simplement que tel n'est pas mon point de vue! Ce qui déclenche un 
éclat de rire général... J'interviens très spontanément dans les discussions. On respire ici un air de liberté et d'aisance que je n'ai jamais ressenti ailleurs: ni en France, ni en Allemagne.

Liberté à l'image de l'océan en bordure duquel je me promène. Il est 18 heures. Il fait nuit. On entend fortement le flux et le reflux. On sent l'air marin salé. Le vent souffle. On sent l'immensité de l'horizon, la Terre entière. Je reste là un long moment, à contempler cette infinité, cette spontanéité qui m'assaillent et me réjouissent. Si les Etats-Unis sont cela, je ne peux que refuser tout anti-américanisme...

19 novembre: journée de détente. La soirée d'hier dans un restaurant très luxueux a été délicieuse. W. Mc Kenna, T. Nenon et K. Held sont de parfaits gentlemen: la courtoisie est leur qualité fondamentale. K. Held est sans doute le plus paternel, T. Nenon le plus séduisant. Quant à W. Mc Kenna, il est la bonté même. Sur la plage, autour de minuit, nous dégustons du cognac en observant le ciel et les étoiles, sur fond d'océan grondant.

Ce matin, en compagnie de K. Held, de R. Bruzina et de Mary Jane Larrabee, baignades, promenades le long de l'océan à la recherche de coquillages. J'en découvre un très beau, nous nous baignons dans les rouleaux.

K. Held nous raconte la Grèce. Il est amoureux de ce pays. Je lui raconte la Turquie: il se montre intrigué...

Lester Embree m'a ramenée chez lui, et me fait part de son projet de Colloque sur le thème Phenomenological Politics. Il ne souhaite ni analyses textuelles, ni dérivations régionales du politique à partir de la phénoménologie transcendantale comme science princeps. Je lui propose de décrire l'attitude politique comme un agir non-agissant libéré par des figures éminemment critiques telles que celles du Bouddha, de Socrate ou du Christ (voire de Mahomet). Je le perçois intéressé mais perplexe... Il parle de sa conception d'une pratique de la phénoménologie plutôt que de sa théorie: je ne peux qu'être d'accord.

Dans la soirée, je lui propose de retirer les feuilles qui encombrent la surface de sa piscine. Activité proprement méditative selon sa fille!

Longue discussion avec Lester hier soir. Je lis d'abord un article de lui à propos des «gender differences in conversational relationships». Au cours du repas, il oppose radicalement phénoménologie transcendantale et psychologie (sociologie) phénoménologique, en identifiant transcendantalité et recherche des fins dernières, en d'autres termes philosophie première ou métaphysique. J'essaie de lui expliquer comment une expérience transcendantale est possible indépendamment de toute 
recherche des fins dernières. C'est alors qu'il parle d'empirisme transcendantal, qu'il m'explique aussi que la nature est animée, qu'il plaide pour un animisme généralisé... La table elle-même a un esprit, finit-il par lâcher...

20 novembre: les «aligators» sont parmi nous. Lester m'emmène dans la réserve naturelle (Wildlife Refuge) des Ever Glades. Je ne peux m'empêcher de penser à la scène de Brave New World... Une fois sur place, cependant, le calme de l'endroit, la beauté des oiseaux prennent le dessus. Nous cheminons parmi les herbes et les marais, en quête d'aligators. A force de scruter la surface de l'eau, c'est un, puis deux, puis trois qui nous montrent le bout de leur nez. Je peux à présent rentrer en France. L'essentiel m'a été donné.

18 heures: en vol. Miami-Paris: 7385 kilomètres. A l'aller, on projeta Nelly et Monsieur Arnauld, de Claude Sautet, plein de finesse et de délicatesse, à l'image de mon état d'âme fragile; au retour, Les Mille, ce film qui narre le sauvetage in extremis d'intellectuels allemands réfugiés en France en 1940, et fuyant devant l'envahisseur nazi. Je rentre des Etats-Unis. Les longues conversations avec Lester Embree m'ont convaincue de la nécessité d'agir, d'une manière ou d'une autre. Comment? Je ne sais, mais ce que je sais, c'est qu'il me faudra intervenir, à un moment donné. Je sais aussi que I'heure n'est pas encore venue, que je ne suis pas mûre pour cela, mais je sens confusément que là est mon destin: non-agir sur le monde pour le parachever. C'est là que l'attitude du phénoménologue devient une pratique à part entière, c'est-à-dire une attitude essentiellement politique.

Dans la nuit: cette communauté en vol, tendue vers et portée par un unique destin, fragile et grégaire...

Mardi 21 novembre, 8 heures du matin: avec le décalage horaire, la nuit fut «blanche». Etrange sensation d'ubiquité: l'ici et le là-bas se bousculent.

Sur le répondeur, je trouve le message d'Emmanuel Falque: Constantin est né samedi 18 novembre à minuit, au «moment» même où, de l'autre côté de l'océan, je contemplais notre origine marine tumultueuse. Je me remémore alors cette parole de Lester juste avant mon départ: «You look very young, but you are very old».

S'est-il passé une journée sans que vous ne connaissiez au moins une fois cet état de trouble, insigne ou banal, c'est selon, où vous vous êtes senti soudain intrigué, perplexe, déconcerté, ébloui, éberlué, ravi, abasourdi, émerveillé, sidéré, étonné, stupéfait, choqué, ou bien effondré? Où vous vous êtes exclamé, 
où vous avez explosé de joie, où vous avez hurlé de colère, où vous avez froncé les sourcils, songeur ou soucieux?

Je sursaute lorsque la porte claque. Je tressaille à la vue d'un rat mort sur l'escalier que je monte quatre à quatre me sachant en retard pour le dîner. J'ouvre grands les yeux à la vue d'un parapente dans le ciel de montagne, d'un arc-en-ciel derrière la vitre du train. J'écoute, le regard fixe, un ami cher au café me raconter qu'il va mourir d'un cancer du foie: je le vois faire le geste d'un doigt qui trébuche et qui tombe de l'autre côté.

Moi qui viens de recevoir ce matin un coup de téléphone m'annonçant que mon père est tombé au réveil et se trouve actuellement dans le coma à I'hôpital de la Salpêtrière, je m'exclame, encore sous le choc: «non, ce n'est pas possible!» A l'instant où j'entends la voix de ma sœur, je suis à cent lieux de m'imaginer qu'elle va m'annoncer sa mort et, les effets immédiats de cette nouvelle, c'est ma voix qui tremble, mon estomac qui se contracte, les larmes qui me viennent et des émotions mélangées de douleur, de tristesse, de regret de n'avoir pas été davantage avec lui ces derniers temps, puis, très vite, ce sont des questions, c'est de l'incompréhension, de la colère voire de la révolte. Et un air de scandale dans la voix: «comment a-t-il pu tomber? Qu'est-ce qui s'est passé? Pourquoi n'a-t-il pas été ranimé?»

Et, pourtant, à la question de savoir si I'on peut ne pas être surpris.e, un logicien répondra spontanément: «oui, bien sûr, la surprise, ça n'existe pas!» Car, en logique, stricto sensu, il n'y a pas de surprise: tout est cohérent, déterminé d'avance, prévisible, il n'y a pas d'incertitudes ni d'équivoques, pas d'aléatoire ni de hasard, tout est... logique! Ainsi, lorsque le mari d'une logicienne qui vient d'accoucher l'appelle au téléphone et lui demande: «alors, c'est un garçon ou une fille?» Celle-ci, en bonne logicienne, lui répond: «oui!» C'est affirmatif, c'est sans surprise, c'est bien «un garçon ou une fille». Même si, pour le mari, qui n'est pas logicien, cela ne répond pas du tout à son attente ni à sa question. La réponse de sa femme produira en lui de la perplexité, voire de I'irritation. Ou, s'il est d'un naturel flegmatique et connaît bien sa tendre et chère, il partira d'un grand éclat de rire de... surprise! Moi qui ne suis pas toujours «logique» loin s'en faut, moi qui voudrais tellement savoir, dans cette histoire de la logicienne, de quel sexe est l'enfant qu'elle vient d'avoir, moi qui suis, comme le mari, déçue, frustrée, ou bien en colère contre ces logiciens qui jamais ne sont surpris, qui toujours ont prévu ce qui va arriver, qui toujours ont une réponse 
rationnelle à tout, moi qui me décide parfois sur un coup de tête à aller me baigner alors que j'avais dit que je n'irai pas, qui mange un carré de chocolat alors que je me l'étais interdit l'instant d'avant, comment pourrais-je ne pas être surprise? Comment pourrais-je réduire ou même éviter ce tressaillement, ce léger décrochage de mon estomac qui me prend si souvent, à la vue d'une personne aimée ou détestée qui apparaît contre toute attente dans mon champ de vision, ou bien ce gonflement imperceptible de ma cage thoracique juste après? Comment pourrais-je tout programmer? Moi qui suis susceptible, à chaque instant, de déprogrammer ce que j'avais projeté initialement! D'ailleurs, est-ce bien «raisonnable» de chercher à éviter les surprises? De les minorer? Quelle est cette pulsion de contrôle qui m'habite alors? Ou, à l'inverse (mais n'est-ce pas la même chose?), quelle est cette anxiété devant l'inconnu, le nouveau, devant ce que je ne maîtrise pas, qui pourrait me pousser à chercher à tout prévoir (sans d'ailleurs y parvenir)? Cette difficulté devant la vie qui, elle, ne choisit pas, ne contrôle rien, me jette sans cesse de la réalité imprévue à la figure et me souffle: «vas-y, fais avec, adapte-toi, cherche à comprendre, accueille, refuse, ou bien... Amuse-t-en...!»

Bien sûr, je peux ne pas aimer les surprises (peut-être parce que j'en ai vécu de trop mauvaises, ou que la vie m'a mise en danger trop souvent), ou bien, à l'inverse, je peux les adorer... Toujours est-il que j'y suis possiblement confrontée (sinon soumise) à chaque instant.

Plus surprenant, l'état de surprise est si présent en moi, il m’habite tant que, si je fais attention à moi-même ne serait-ce qu'un instant, je me rends compte que je ne cesse de me surprendre: ne suis-je pas l'instant d'après différente de ce que j'étais? D'ailleurs, la moindre idée qui me vient: «tiens, la deuxième partie de mon roman, ça va être la prise de conscience d'un bout de son passé que I'héroïne croyait englouti pour toujours!!» Ou bien: je sors les poubelles et je croise un cousin qui me propose de venir prendre le café. Ou encore: je suis en train de lire et le téléphone sonne: «j'apprends que mon neveu vient d'avoir une petite fille!» A chaque instant, quelque chose arrive qui me fait bouger (ou pas), un événement, même infime, est une rencontre avec moi-même, et surtout avec ce quelqu'un en moi qui n'est déjà plus tout à fait le moi que j'étais l'instant d'avant: «waouh! C'est moi qui ai fait ça? Exemple: j'ai fait hier une descente en parapente du haut du Mont Chéri! Je ne m'en serais jamais cru capable!» Voilà 
qui suppose un écart même ténu entre ce que je suis et ce que je crois ou bien me représente être. Cela, on l'expérimente très souvent aussi: je me lève le matin avec une grosse flegme et je me retrouve à la fin de la journée à avoir fait un nombre incalculable de choses, des courses, écrit un article, emmené ma fille au cinéma, préparé un repas, appelé un collègue pour un problème à mon bureau, à I'Université, etc. Je me regarde dans la glace le soir, et je me dis, avec un grand sourire: «c'est toi qui a fait tout cela: tu me surprendras toujours!» Bref, il y a très souvent ce quelqu'un en moi-même, comme un léger témoin, qui me fait faire ou voir les choses autrement, qui me fait (parfois) prendre du recul sur ce que j'ai pensé ou dit l'instant d'avant, sur ce que je croyais ne jamais pouvoir accepter, assumer, intégrer (un deuil, une séparation...).

Il y a des milliers de surprises dans une journée, et on ne sait jamais le matin de quoi ce jour sera fait. En dépit de tout ce que l'on a bien pu prévoir de faire... Ces mini-surprises tissent notre vie à chaque instant. On n'y prête pas garde, on les vit, elles passent, elles collent à la poussée intime de notre être et nous font vivre, croître aussi, à notre insu. Alors, quand cessent les surprises, est-ce que cela veut dire qu'on est sur le point de mourir, ou bien, a minima, qu'on est en dépression, parce que I'horizon est bouché, parce notre liberté de ne rien déterminer d'avance, de tout laisser ouvert s'est retirée dans les replis nostalgiques régressifs de notre passé? Ou bien est-ce parce que l'on n'a plus rien à attendre? Plus d'espoir? Mais aussi plus de crainte? Que I'on est apaisé? Dans un quasi-état de méditation profonde? Les surprises, cependant, ce sont peut-être aussi (tout autant) des illusions utiles qui nous font vivre. Est-ce donc que, si j'arrivais à être moins surprise: en étant plus logique? Plus attentive? Plus zen? -Je vivrai mieux? Ou bien moins bien?

Et d'ailleurs: d'où vient que les langues, littéraires ou ordinaires, fourmillent de locutions qui disent cette situation inépuisable de réceptivité, d'ouverture inédite à ce qui vient, de stupeur aussi, et l'expriment par des exclamations, des interjections, des interrogations: «quelle ne fut pas ma surprise quand je t'ai aperçue au coin de la rue?». «J'ai été prise de court quand tu m'as annoncé que tu partais vivre au Tibet: je ne m'y attendais vraiment pas! Quelle surprise!». Est-ce que cela veut dire que les langues, voire le langage, ne sont pas seulement logiques, voire pas logiques du tout? Qu'elles traduisent notre état souvent 
incertain de ce qui va avoir lieu l'instant d'après, entre euphorie et désespoir... Qu'elles sont chargées d'intuitions, de corps et de sang?

Les surprises, donc, sont omniprésentes dans nos vies. Pour le meilleur: au quotidien, ce sont des sortes de mini-sursauts, des micro-prises de conscience, des légers décrochages, ou bien, ce sont des exceptions à la règle qui nous font sortir de l'ordre, de la routine, de l'habitude ou de l'ennui. Nous avons tous connu ce temps étale des vacances, où rien ne se passe, où rien n'est prévu... Tout est ouvert. Pouvoir ne rien faire, enfin! Et puis, ça ne manque pas, la vie reprend le dessus, on se rassemble. Il y a quelque chose qui survient, toujours. Quelque chose d'inédit qui te fait vibrer: tu n'avais pas prévu d'aller voir le feu d'artifices ce soir. Une proposition, chouette, ça ne se refuse pas. Ou bien ça te dérange et tu préfères rester tranquille à la maison avec un bon film. Dans tous les cas, on a toujours le choix face à un petit miracle: y adhérer ou le décliner. Ou, pour le pire: une guérison, une catastrophe ferroviaire, une guerre. C'est différent, ça te tombe dessus et tu en subis les effets, affects, somatisation, flux de pensées, associations, interrogations, trauma, etc.

Cela a à voir, sans doute, avec l'intensité de la surprise, qu'elle soit bonne ou non. Si le degré est extrême, la marge de manœuvre pour l'accueillir au moment même, pour délibérer une fraction de seconde en soi-même, pour apprécier comment l'accepter, I'accueillir ou la refuser, est beaucoup plus faible voire inexistant. J'entends à la radio que 200 personnes sont mortes dans un accident d'avion au dessus de l'océan atlantique! L'intensité et I'objectivité de la nouvelle me sidèrent ne serait-ce qu'un millième de seconde, avant que le processus pour tenter de comprendre, pour rationaliser se mette en branle. Ou bien, je suis prise d'admiration devant ce tableau de Egon Schiele, ce jeune garçon aux grands yeux verts, que je n'avais jamais vu. Un saisissement interne fait son chemin et me conduit à exprimer ma surprise, avec cette conscience qui affleure. Ou bien: «tiens, regarde cette peinture de Chirico, elle est vraiment curieuse! Je n'avais jamais vu des têtes pareilles en forme de ballons de rugby! C'est bizarre, non?». Les surprises, qu'elles soient infimes ou énormes, se glissent au détour du moindre de nos actes: «j'ai regardé le premier épisode de Game of Thrones, je l'ai trouvé étrange, je ne m'attendais pas à une ambiance aussi crue, si peu romantique...»; de nos paroles les plus anodines: «tu verras, si tu vas voir ce spectacle de chevaux du cirque Alexis Grus, tu vas avoir la surprise de ta vie!». Bref, les surprises font de nous des sujets sans prétention, 
toujours ouverts, des sujets qui prennent le risque de ne pas savoir, le risque de se perdre, d'être détournés de nos assurances, d'être effondrés en notre centre le plus intime, pétrifiés, abasourdis ou bien vivifiés, galvanisés, c'est selon. La surprise, petite ou grande, me confronte à l'incompréhensible et impose en moi l'absence de sens, quitte à susciter ravissement, vertige, désespoir.

Ce qui est frappant, c'est que la surprise n'est pas un concept philosophique. C'est peut-être cela, la plus belle surprise. A moins qu'il y ait là quelque chose à interroger. Quelque chose d'incongru. D'ailleurs, en parler au singulier, au nominatif, comme si elle était le sujet d'une phrase constative, comme si elle était un concept ainsi que je l'ai supposé dans le titre de l'ouvrage, bien sûr, on peut le faire, mais c'est la perdre en partie, car «la» surprise, ça n'existe pas. L'être surpris, c'est un être situé, ici et maintenant, c'est le sujet d'une exclamation, d'une interrogation, c'est un être-processus qui se dit par un verbe autant que par un nom et, souvent, au passif de son participe passé: «ça m’a tellement surprise!». Justement, quand elle paraît, je n'y suis plus, et je réapparais juste après, une fois qu'elle est passée. Il y a eu dirait-on un blanc d'antenne, une syncope, comme lorsque le cœur, une fraction de seconde, s'arrête de battre. Comme si être surprise ne pouvait se dire qu'au tout juste passé: un 'vécu', au sens littéral de quelque chose qui a déjà eu lieu, et qui, au moment même, est un non-temps, un non-lieu, une non-expérience. «Ça me surprend que tu dises que tu aimes ce film, ça ne te ressemble pas!». Quand je m'exclame, l'état de surprise qui me traverse a déjà commencé avant que je m'en rende compte, même s'il peut durer encore au moment de le dire, et perdurer au delà de mon exclamation, comme un filament psychique diffus ou bien obsédant.

Bien sûr, on a pu dire cela du temps également, de l'instant présent, qui est déjà passé au moment où je le nomme, ou bien, a fortiori, au moment où je I'identifie. A ceci près que je ne cesse jamais d'être surprise: cela se réactive à chaque fois à l'identique, avec une qualité d'intensité toujours aussi prégnante quoique nouvelle, alors que l'instant qui a eu lieu une fois est perdu à jamais. Certes, je peux m'en souvenir, je peux l'évoquer avec la vivacité et la finesse vertigineuse du quasi-revécu, mais mon souvenir de ce moment douloureux où j'ai appris la mort de cet ami si proche ne sera jamais identique en intensité à ce moment là: Nicodème, tu ne peux pas revivre à l'identique ce qui a déjà été vécu. 
L'intensité du souvenir peut être extrême, plus intense même, lorsque par exemple le moment aura été traumatique (une bombe qui explose dans la station du métro Saint Michel dans laquelle je m'apprête à m'engouffrer). Cependant, même alors, le trauma qui revient en force au centuple lorsque je m'approche à nouveau de cette bouche de métro, revivifié dans des aspects isolés, décalés voire déformés (le panneau qui indique I'horaire d'arrivée du train et télescope en moi cette vision avant l'incident, un message sonore «attentifs ensemble», qui résonne en moi comme une sirène stridente annonçant l'incidentcatastrophe), ce n'est pas le moment même du trauma, mais seulement un moment du processus traumatique.

La surprise, elle, se réactive toujours à l'identique dans sa structure, en dépit de son contenu toujours nouveau: j'ouvre la porte de la maison à la volée, tu es cachée derrière, tu cries « waouh! » et je sursaute à chaque fois avec la même qualité de présence. J'ai beau le savoir, ou l'avoir su, le pressentir, sur le coup, c'est toujours une première! Il y a là quelque chose qui a à voir avec un autre état d'être, l'attention, qui toujours se perd, fragile, instable, ténue, qui toujours se réactive, restaurée, et à l'identique: je peux redevenir attentifve avec la même intensité, après avoir été perdue dans mes pensées l'instant d'avant. Je fixais un enfant en roller, mon amie me parle, je lui réponds, il m'a distraite le temps d'une parole, je regarde à nouveau l'enfant, il est tombé entre temps, je ne l'ai pas vu, et je suis à nouveau attentif.ve à lui comme alors, avec la même qualité. Le temps, lui, file et coule, irréversible et entropique.

Etre surprise, comme être attentifve, c'est connaître cette sensation intime, fugace. Tout à la fois, elle se maintient peu, elle s'évapore aussi vite qu'elle est venue, mais elle est marquante, intense: je m'en souviens, je peux réactiver cet instant qui a suscité en moi un léger décrochage, qui a contrasté avec l'instant précédent, ou bien avec un temps continu où rien semble-t-il ne se différenciait du moment précédent. Je sursaute en entendant un verre qui bascule dans l'évier rempli de vaisselle sale, cela fait partie de moi, je m'en souviendrai. Je fixe la branche du tilleul qui oscille sous le vent, cela s'imprime en moi, c'est déposé dans ma mémoire. Bien sûr, ces sédiments sont souvent involontaires, organiques, liés à une sensation, auditive (le bruit du verre qui se brise), visuellemotrice (le bruissement de la feuille). Ils n'en restent pas moins gravés en moi. 
La question qui naît confusément dans mon esprit pourrait être: est-ce que, si je suis suffisamment attentifve (ce que je peux être parfois...), à défaut d'être logique (ce que je ne suis pas), si je cultive un état méditatif d'ouverture (ce à quoi je m'efforce), je pourrais parvenir, sinon à supprimer toute forme de surprise (ce serait peut-être un peu radical, abstrait, idéal...), du moins à diminuer son impact perturbant, le ralentir, la faire bifurquer? Si je fixe avec toute la concentration dont je suis capable la branche de tilleul où s'imprime le vent, vais-je pouvoir suivre son mouvement et anticiper le mouvement du vent I'instant d'après?

Mais cela voudrait dire qu'on a intérêt à être moins surprise, parce que la surprise, ce serait ce qui dérange, ce qui trouble, ce qui fait mal, comme une crise d'épilepsie que l'on fera bifurquer en en repérant les signes avant-coureurs. On dit que le cerveau, spontanément, minimise les surprises, autant d'effets perturbants qui entravent sa bonne marche: il y a un système d'autorégulation qui permet d'éviter les «bugs». Est-ce à dire que les surprises ne seraient que négatives, qu'elles feraient obstacle au fonctionnement neuronal? Cette vérité, incontestable, scientifique, est-elle pour autant complète? Et s'il y avait un bénéfice du bug, du décrochage, qui serait une sorte de ressort à la plasticité du fonctionnement de notre cerveau, à sa régénération? On dit aussi que la surprise a pour fonction de m'aider à m'adapter à des situations nouvelles. A fortiori, l'expérience subjective d'une personne ne témoigne-t-elle pas de moments de surprise qui sont autant d'ouvertures? Des effractions soudaines de sens, des instants de création qui donnent un surcroît d'être, une intensité vitale, là où il y avait I'instant d'avant un sens plus pauvre, un être plus banal?

S'il y a un bénéfice des surprises, peut-être faut-il moins les supprimer voire les diminuer que les cultiver. Sans doute convient-il de ne pas les subir, car, à subir trop, on souffre: on connaît alors moins la surprise que la stupeur, la sidération du choc, exemplairement traumatique. Mais, alors, comment s'y préparer, s'il semble, d'après ce qu'on a vu, que le propre d'une surprise, c'est qu'on ne s'y attend pas, et qu'elle apparaît dans son contraste complet avec l'attente, c'est-à-dire avec l'attention? La surprise tiendrait-elle dans cet oxymoron d'un inattendu objet d'attente, ou encore d'une attente sans attente? A cet égard, que signifie cette expression de la langue qui nous invite par exemple à «préparer une surprise» à un enfant dont c'est l'anniversaire? 
L'enfant qui attend une surprise, à qui son père prépare une surprise, est dans la situation de quelqu'un qui sait que quelque chose va se produire sans savoir ce qui va se produire. Ce savoir est tout sauf une prévision (type loi d'inertie), à la manière du galet que je lance et dont je sais qu'il va tomber dans I'eau. Ce savoir est entièrement ouvert, indéterminé. Il relève cependant de l'assurance qu'il y aura quelque chose. C'est le même type de savoir qui est en jeu lorsque je me prépare à un examen du mieux que je peux, tout en sachant, d'une sorte de savoir du non-savoir, que je ne pourrai anticiper tous les sujets. Quoi que je fasse, je serai surprise, soit que le sujet qui 'tombe' est une de mes impasses: ma surprise sera alors, par défaut, une déception, voire une colère; soit qu'il dépasse toutes mes attentes, comblant mes souhaits au delà de l'espéré: c'est exactement le sujet que j'ai traité il y a deux semaines. Je serai alors surprise par excès de satisfaction: comblée au delà du désirable, émerveillée, subjuguée. Jusque dans le cas où le sujet donné à l'examen correspond exactement à ce que j'espérais en l'ayant anticipé: cela est suffisamment rare pour que j'en sois, cette fois encore, surprise, ébahie devant l'adéquation parfaite et ma foi miraculeuse entre mon attente et le sujet donné.

Bref, quoi que je fasse, surprise il y a.

Ce que je peux faire de mieux, c'est me préparer à l'existence d'une surprise dont le contenu ne peut que m'échapper. Mon seul savoir tient dans le fait que je m'attends à présent à être surprise. Je peux cultiver cette attitude où je vais m'habituer à me «laisser» surprendre, mon attention consistant dans cette ouverture complète à (tout) ce qui peut se produire. On est loin de l'attention qui consiste à se fixer sur un point, et qui fait de tous les autres points une source de distraction, déclenchant par contraste une surprise née de l'excès de concentration...

Dira-t-on qu'il n'y a de vraie surprise, de surprise vraiment accueillie que sur le fond d'une attention continue, qui ressemble davantage à une sorte de présence discrète à ce qui peut survenir, d'ouverture accueillante à tout, sans savoir ce qui peut arriver? Cette formulation est paradoxale. Car on dira d'abord, comme nous y invite la question initiale, que je suis bel et bien surprise de voir ma collègue de faculté au bord de la mer: je ne m'attendais pas à la voir ici à ce moment précis. Ma surprise tiendrait alors à ceci que je n'aurai pas été assez en attente de la voir, pas assez réceptif.ve à la possibilité de son apparition, l'attente allant de pair avec une sorte d'attention, de présence à sa venue toujours 
imminente... Mon attention était portée ailleurs, tournée vers mon fils qui joue avec ses voitures, ou bien absorbée dans la lecture du dernier roman de Musso, et c'est pourquoi j'ai été surprise en entendant ce bruit de pas pourtant si caractéristique qui est le sien sur les graviers de la cour.

Cette surprise, qui est liée à un manque d'attention, à savoir à une attention détournée, déviée, captivée, à une attention placée ailleurs, est une surprise qui s'impose à moi. Je la subis, je suis passif.ve à son égard. C'est l'expérience courante que l'on fait, et pour laquelle les exemples ne manquent pas: «tu m'as prise en flagrant délit» dira ma fille en train de dérober du chocolat dans le placard. Toute à la recherche de sa plaque de chocolat, elle ne m'a pas entendue arriver derrière elle! Nous sommes dans la voiture en train de rouler, chacun attentif, le conducteur à la route, le passager lisant la carte, les jeunes sur les sièges arrière regardant le paysage, écoutant de la musique ou lisant: quelle surprise! Un chevreuil traverse inopinément la route de campagne juste devant les phares de la voiture à la nuit tombée, qui pile sec. Personne ne l'a vu arriver, quelle que soit notre attention ou notre distraction. Le passager qui regarde par la fenêtre aurait pu le voir, mais il regardait ailleurs, ou regardait sans voir; le conducteur, focalisé sur la route, n'avait pas une vision panoramique, ouverte aux abords de la forêt; les autres passagers, absorbés, qui dans leur lecture, qui dans leur musique, auraient pu entendre ou voir autrement, mais, non, tous sont surpris au moment où le chevreuil déboule sur la route!

Ainsi, être surprise rejette hors de soi, de par le mouvement brusque par là suscité, la possibilité d'une attention qui en serait l'annonce voire la préparation. Par là, la surprise nous expulse hors de nous-mêmes.

Ce livre voudrait dessiner une carte des surprises. Comme des menus qui déroulent entrées plats desserts, on déroulera attentions, crises-ruptures, émotions... On montrera les écarts de sens entre, d'une part, différentes entréesattention: absence, distraction, focalisation, négligence, ouverture, attente, imminence; d'autre part, divers plats-ruptures: sursaut, perplexité, prise de conscience, tressaillement, sidération, stupeur, saisissement, stupéfaction; de multiples desserts-émotions, enfin: soulagement, étonnement, émerveillement, ou bien rumination, peur, colère, dégoût, joie ou tristesse. On cherchera à rendre compte du processus-repas qui conduit à ces états ou à ces processus affectifs 
et à ce qui en résulte en termes d'effets immédiats. C'est là qu'on fera apparaître la dynamique qui relie la surprise dans ces différentes formes avec l'attention selon ses divers régimes, concentration, veille, réceptivité, absorption, vigilance, capture, et l'émotion dans ses valences plurielles. Telle est la proposition du présent ouvrage: manifester la dynamique interne de la surprise dans ses modalités de relation saillantes avec l'attention et l'émotion, ses deux structures internes.

J'entends une question: pourquoi cela? Quel enjeu crucial y a-t-il donc à cela? On a pu percevoir, à la lecture des pages qui précèdent, combien surprise, attention et émotion font tellement partie de notre quotidien qu'on les tient spontanément pour acquises: on n'interroge pas leur importance pour notre vie, ni pour notre humanité. C'est ce que voudrait faire ce livre. Nous amener à nous rendre compte que, avec la surprise, il y va de la vie et de notre vie d'être humain, de notre être-nous dans notre être hors-de-nous à chaque instant de notre vie: la surprise, c'est l'étoffe non-sue de notre état d'être humain le plus profond. La surprise nous fait sortir de nous.

Ce livre n'a pas pour but de construire un concept exclusif de surprise, mais de tracer sur une carte certains de ses chemins de randonnée. J'y forme l'espoir que le vivant humain que nous sommes pourra peut-être mieux s'orienter dans le parcours qu'est sa vie, en empruntant ces différents chemins. Au stade actuel de mon exploration du phénomène de la surprise, j'aperçois sept chemins qui sont autant de micro-modèles de la dynamique attention-surprise-émotion. En attendant que lecteur, lectrice, tu me surprennes en m'en faisant découvrir un autre! En fait, il y en a bien encore un huitième, mais je vous laisse la surprise de le découvrir...

Ces micro-modèles ne s'opposent pas ni ne sont situés sur une échelle de valeurs. Plus simplement, ils correspondent à des modes d'être différents que chacune d'entre nous rencontre, dont elle ou il emprunte le chemin, ou qu'elle ou il peut connaître à divers moments de sa vie, mais aussi, couramment, de sa journée.

(1) Une de nos attitudes les plus ordinaires, c'est l'attention absorbée (dans un livre), captivée (par un film), hyper-focalisée (dans une raisonnement). Il y a, explosion, klaxon, sonnerie de téléphone, un ami qui se présente soudain devant moi. C'est la surprise-sursaut, sorte de réflexe, l'attention ayant été 
détournée, puis, parfois, réorientée, ou délitée, perdue. Processus à la fois courant et complexe dont j'explorerai le décours dans le premier chapitre de la III ème Partie de l'ouvrage.

(2) Une telle attention est paradoxale, à la fois passive (je suis absorbée, captivée) et éminemment active (je suis hyper-focalisée, concentrée). Dans ce dernier sens, elle s'apparente à un contrôle qui expulse hors d'elle-même tout ce qui pourrait la déstabiliser (jusqu'à générer parfois une tendance pathogène), et veut faire triompher une logique rationnelle. Cette attention-contrôle, envahissante, anticipative, programmatrice, à force d'expulser la surprise hors d'elle, peut aller jusqu'à la faire disparaître, ou du moins la réduire, la banaliser, la minimiser. Dans nos vies contemporaines (occidentales?), une telle tendance est très formatée, et il sera intéressant d'en repérer les accents et les points problématiques, voire pathologiques.

(3) Mais la surprise éclate d'autant plus qu'elle a été incluse dans la représentation que j'ai de ce qui peut arriver. Ainsi, loin de diminuer voire de disparaître comme en logique, la surprise dans les relations humaines montrent que la vie est le plus souvent illogique. En logique ou dans notre cerveau, il y a une volonté de prévision, de contrôle d'autrui dans ma relation avec lui, mais il ne s'agit pas là d'une simple programmation objective. Cela inclut aussi du désir et du pouvoir: la prévision se fait projection, faite de crainte et d'espoir, d'appréhension et d'euphorie. Je voudrais tant qu'autrui soit comme je veux qu'il soit, et non comme il est.

Or, autrui est comme il est. Il ne peut que me surprendre: il ne correspond en fait jamais à ma représentation.

La surprise se fait méprise, génère des émotions relationnelles de déception, mais elle peut aussi être com-prise si autrui et moi-même sommes complices. Le plus souvent, les interactions intersubjectives fourmillent de malentendus, de quiproquos, et font naître des émotions d'amertume, mais elles peuvent aussi parfois engendrer de la connivence voire, moment de grâce rare, un sentiment d'harmonie.

Ces trois dynamiques ont une caractéristique commune: elles reposent sur le schème d'une exclusion réciproque de l'attention et de la surprise, soit subie, soit voulue, soit projetée-incluse, et elles génèrent une émotionnalité potentiellement négative voire pathogène. Car, au fond, subir, contrôler ou projeter sont des facettes différentes de la même attention, des modalités de 
mon expérience passive-active, qui coïncident aussi à travers ce qu'elles engendrent de souffrance, de mal-être, ou de malentendu.

(4) Si le subir devient mon mode d'être permanent, si le contrôle s'impose, si la projection me hante, il y a fort à parier que l'attention se fasse rigidité, obsession, et que la surprise devienne incessante et déstabilisatrice. La conversion pathologique de l'attention est aussi une conversion pathologique de la surprise. Elle va de la dépression, où la surprise s'émousse, à la schizophrénie, où elle s'exalte. L'émotion devient envahissante, fait reculer l'attention et perturbe à l'excès les régulations internes du vivant.

Aussi, à mesure, je vais, ou bien on va m'aider à développer des stratégies pour faire fructifier des ressources destinées à moins subir, mais aussi, de ce fait, c'est tout un, je contrôlerai moins et je projetterai mieux.

Une des clés tient donc dans le fait de cultiver une forme d'ouverture à la situation qui vient. Cet accueil peut prendre deux formes différentes: disponibilité ou fulgurance. Dans les deux cas, l'ouverture suppose un exercice, un entraînement, un jardinage: ce n'est pas une attitude spontanée, standard ou naturelle. Elle exige un effort, et cela prend du temps.

(5) Une modalité de cette ouverture se donne comme une attention-attente, placée sous le signe de la réceptivité. Ni passif, ni actif, inscrit ailleurs, dans une plus riche complexité, le sujet réceptif est acteur de ce qui arrive (il ne subit pas), sans pour autant tout maîtriser: il se tient là, dans une attente éveillée. Il y a une patience qui nous place dans une durée indéterminée, mais invite à garder I'initiative: I'action y est posture de veille. Du coup, la surprise qui survient, attendue en structure, cultivée, sera goûtée comme telle, dans son contenu irréductible: on « se laissera » surprendre.

(6) Une autre modalité de l'ouverture en jeu dans la dynamique de l'intégration mutuelle de l'attention et de la surprise répond à une attentioninstant, du type de la saisie du moment présent tel qu'il se présente lui-même. Il y a un art de cultiver l'instant dans son intensité et sa fulgurance, et d'en apprécier la qualité propre, fraîcheur ou vertige. A cette attention instantanée, sans réserve, sans sédimentation ou stockage répond une surprise-nouveauté, toujours différente, jamais anticipable: irréductible. 
Les trois premiers modèles de l'antinomie exclusive de l'attention et de la surprise rehaussaient la première au détriment de la seconde. A terme, la surprise s'extériorise, s'efface ou se trouve pré-incluse, respectivement au quotidien, en logique ou dans la représentation d'autrui. Ces trois modèles peuvent engendrer des pathologies où l'émotionnel envahit, et l'attention, et la surprise. Les trois derniers modèles d'intégration_interne que j'esquisse produisent a contrario un retrait de l'attention au profit de la surprise. Ils génèrent une figure de l'émotion placée sous le signe de l'excès, positif ou négatif, voire conjoignant les deux au cœur d'une tension oxymorique qui trouve son acmé dans la contemplation méditative ou mystique, dans l'ouverture esthétique, dans l'attitude éthique enfin.

(7) C'est là que je puis devenir tout entier surprise. Être une surprise pour moi-même: une énigme. La surprise ne sera plus un objet que je prépare pour quelqu'un, ni un effet résultant de mon manque d'attention ou de mon hyperfocalisation. La surprise ne sera pas non plus un instant qui fulgure ou une réalité que j'accueille. Moi et la surprise, c'est tout un, moi qui suis toujours nouvelle, nouveau à moi-même, qui vis de micro-ruptures, d'écarts internes. Je suis me surprenant, dans cette altérité interne qui me forge. Je suis le sujet de la surprise.

Je suis le soi surprenant:

ou bien, tout simplement, le surprenant plutôt que l'être surpris.

Je suis le surprenant, c'est-à-dire, il y a le surprenant, et ils sont multiples.

Du coup, il y a des 'surprises' collectives. D'où le huitième et dernier chapitre, qui aura aussi pu être le premier...

PS: Lester, je t'offre l'Introduction de ce livre à venir sur la surprise que j'écris en ce moment avec peine. Il est si malaisé d'écrire en philosophie en se désencombrant des concepts et de la technicité des analyses: ce fut toute ta vie, elle me poursuit... 